Sādhanā Vol. 39, Part 1, February 2014, pp. 81-95. (C) Indian Academy of Sciences

\title{
Certain investigations on the reduction of side lobe level of an uniform linear antenna array using biogeography based optimization technique with sinusoidal migration model and simplified-BBO
}

\author{
T S JEYALI LASEETHA ${ }^{1, *}$ and R SUKANESH ${ }^{2}$ \\ ${ }^{1}$ Department of Electronics and Communication Engineering, \\ Rajiv Gandhi College of Engineering, Anna Unviersity, \\ Chennai, 628 851, India \\ ${ }^{2}$ Department of Electronics and Communication Engineering, \\ Thiagarajar College of Engineering, Madurai, 625 015, India \\ e-mail: laseetha@gmail.com; drsukanesh@gmail.com
}

MS received 27 July 2011; revised 18 May 2012; accepted 5 June 2013

\begin{abstract}
In this paper, we propose biogeography based optimization technique, with linear and sinusoidal migration models and simplified biogeography based optimization (S-BBO), for uniformly spaced linear antenna array synthesis to maximize the reduction of side lobe level (SLL). This paper explores biogeography theory. It generalizes two migration models in BBO namely, linear migration model and sinusoidal migration model. The performance of SLL reduction in ULA is investigated. Our performance study shows that among the two, sinusoidal migration model is a promising candidate for optimization. In our work, simplified - BBO algorithm is also deployed. This determines an optimum set value for amplitude excitations of antenna array elements that generate a radiation pattern with maximum side lobe level reduction. Our detailed investigation also shows that sinusoidal migration model of BBO performs better compared to the other evolutionary algorithms discussed in this paper.
\end{abstract}

Keywords. Biogeography based optimization; linear migration model; sinusoidal migration model; simplified-BBO; linear antenna array synthesis; side lobe level reduction.

\section{Introduction}

\subsection{Linear antenna array synthesis}

Antenna array pattern synthesis is a technique of generating radiation pattern of desired shape and characteristics. This can be achieved by determining the optimum current excitations to be

*For correspondence 
applied to the antenna array elements referred as weight vector. Weight vector, in general is a complex quantity consisting of amplitude and phase. The radiation pattern can be controlled by three different possible combinations of weight vector parameters; (i) amplitude only control, (ii) phase only control, (iii) complex weight control. The two different purposes are gratified using optimum weights - first, side lobe level reduction (SLL) and second, null formation. In our investigation, we are concerned with side lobe level reduction using optimum weights with phase only control. SLL reduction accounts for saving power and minimizing interference to desired signal which is along the Main Response Axis (MRA) of broadside array. The side lobe level less than -13.5 to $-20 \mathrm{~dB}$ with respect to main lobe's peak, is the accepted generalized result. Achieving SLL below $-20 \mathrm{~dB}$ is the main aim of the research work being carried out these days. It is the prime focus of this investigation to achieve SLL below $-40 \mathrm{~dB}$.

\subsection{Model of an antenna array and the problem formulation}

In this work, the antenna elements are assumed to be uniformly spaced, in a straight line along the $\mathrm{y}$-axis, and $\mathrm{N}$ is the total number of elements. The physical separation distance is $d$, and the wave number of the carrier signal is $k=2 \pi / \lambda$, where $\lambda$ is the wavelength. The product $k d$ is the separation between the antennas in radians. When $k d$ is equal to $\pi$ ( or $d=\lambda / 2$ ), the antenna array has maximum gain with the greatest angular accuracy with no side lobes. The phase shift between the antennas experienced by the plane wave is $k d \cos \theta$ and $\theta$ is measured from the $\mathrm{y}$-axis, starting from the first antenna, as shown in figure 1.

Weights can be applied to the individual antenna signals before the array factor (AF) is formed to control the direction and directivity of the main beam. This corresponds to a multiple-inputsingle-output (MISO) system. The total AF is just the sum of the individual signals, given by Balanis (2005) in phasor notation as

$$
A F=\left|\sum_{i=1}^{N} \vec{E}_{i}\right|=\sum_{i=1}^{N} e^{j k_{i}}
$$

The factor $\mathrm{K}=\left(\mathrm{i} * \mathrm{kd} \cos \theta+\beta_{i}\right)$ is the phase difference. Only the magnitude of the AF in any direction is important as the absolute phase has no bearing on the transmitted or received signal. Therefore, only the relative phases of the individual antennas signals are important in calculating the AF. Any signal component that is common to all of the antennas has no effect on the magnitude of the AF. If it is assumed that the antenna elements are symmetric about center of the linear array then in complex notation far field array factor of this array with even number of isotropic elements $(2 \mathrm{~N})$ can be expressed as

$$
A F(\theta)=2 \sum_{i=1}^{N} a_{i} \cos \left(2 \frac{\pi}{\lambda} d_{i} \sin \theta\right) .
$$

The main objective of this work is to find an appropriate set of required element amplitude $a_{i}$ that achieves interference suppression with maximum side lobe level reduction.

To find a set of values which produces the array pattern with nulls at any prescribed direction the algorithm is used to minimize the following cost function (Simon 2008)

$$
\operatorname{Cost}_{S L L}=\sum_{\theta=-90^{\circ}}^{90^{\circ}} W(\theta)\left[B_{o}(\theta)-B_{d}(\theta)\right],
$$

where $B_{o}(\theta)$ is the pattern obtained using our proposed algorithm and $B_{d}(\theta)$ is the pattern desired. Here it is taken to be the Chebychev pattern with SLL of $-40 \mathrm{db}$ and $W(\theta)$ is the weight 


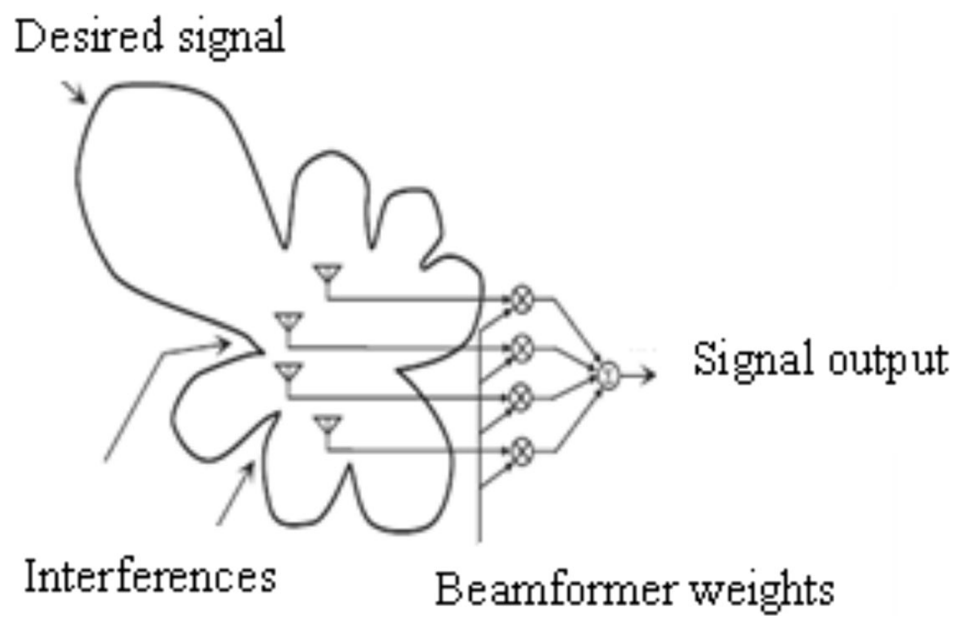

Figure 1. Antenna array.

vector to control the side lobe level in the cost function. The value of cost function is to be selected based on experience and knowledge.

\subsection{Theory of $B B O$}

Biogeography is the science of the distribution of species over space and time. It analyses the existing organisms and their evolution, extinction, and migration. Biogeography was first studied and reported in the work of Alfred Wallace and Charles Darwin. In engineering applications, the evolutionary algorithms including genetic algorithm (GA) (Haupt and Werner 2007), Ant-Colony-Optimization (ACO), Particle Swarm Optimization (PSO) are deployed to find the optimum solutions. Another type of evolutionary algorithm (EA) called biogeography-based optimization (BBO) was recently proposed in the year 2008 by Dan Simon (2011). Mathematical model of biogeography was developed in his paper in terms of factors such as habitat area, immigration rate and emigration rate and describes evolution, extinction and migration of species. He demonstrated its performance on 14 benchmark functions. As mentioned in Ma (2010), this paper failed to present the theory vividly. Dan Simon has also confessed (Simon 2011), that it is much complicated to explain about the probabilistic nature of migration and mutation.

In general, like other EAs, BBO can share information between solutions using migration and mutation. The unique characteristics of BBO are (i) the same set of solution is maintained but improved from generation to generation by migration. (ii) in each generation, emigration and immigration rates are decided based on their fitness.

A habitat is a residing place of many species. The diverse species may be available in abundant and are distributed over the whole habitat. Any species may emigrate to nearby habitat or immigrate into another habitat from a neighbour. The reasons for emigration can be (i) dense population, (ii) limited resources and (iii) natural calamities, etc. The reasons for immigration are (i) to explore new habitat which has sparse population, (ii) to utilize the resources available in abundant. After immigration we may not be sure of whether the immigrant will survive or not. 


\section{BBO based optimization of side lobe level reduction in ULA}

Side lobe level reduction is an optimization problem. The BBO based optimization for an ULA (Singh et al. 2010) depends on the geometry of an antenna and its array factor. In our work, we use uniform linear antenna array, whose array factor is given by (2). The array factor determines the radiation pattern and it depends on the weight vector used. Each weight vector consists of a set of values depending on the dimension of the problem or the number of variables used. Hence weight vector is the candidate solution. The weight vector is tested for its fitness or cost function - minimum SLL. The set of weight vector makes the population. In GA, the weight vector values are represented by genes and the weight vector is represented as chromosome. The population consists of chromosome as the candidate solutions. They survive based on their fitness function or the cost function.

In BBO, weight vector variables are the Suitability Index Variables (SIVs). The set of SIVs is called Habitat. These habitats are tested for minimum side lobe level.

In simple words

Each variable in weight vector, the gene is the SIV

Set of SIV becomes chromosome, the habitat or island

Set of chromosome makes the population, the set of habitat

SLL is the cost function or fitness value of each weight vector

The SIVs are performed with two probabilistic suitability tests namely migration and mutation. Based on the probability, the suitability of the SIVs is assigned some suitability index (SI). High suitability index (HSI) and Low suitability index (LSI) are used to specify the suitability in general in the literature. In linear migration model, the immigration and emigration rates are given by eqn. (4) and (5), respectively.

$$
\begin{gathered}
\lambda_{k}=E K / P, \\
\mu_{k}=I\left(1-\frac{K}{P}\right),
\end{gathered}
$$

where $\mathrm{E}=$ maximum $\lambda, \mathrm{I}=$ maximum $\mu, \mathrm{P}=$ population size, $\mathrm{S}_{\max }=$ size of dataset $=$ Maximum number of species, $S_{\min }=1$. The immigration and emigration curves are straight lines as shown in figure 2, for the case of $E=I$ the linear migration model. Habitat with few species (low HSI, poor solution) have low $\mu$ and high $\lambda$, while habitat with more species $\left(\mathrm{H}^{2} \mathrm{SI}\right.$ good solution) - high $\mu$ and low $\lambda$. The two important concepts of BBO are explained here.

\subsection{Migration}

Any suitability index variable (SIV) of a solution $\mathrm{Si}$ will be selected for modification based on the probability (Pmod) of the immigration rate $\lambda \mathrm{i}$, where Pmod, is an user defined function, known as Habitat Modification Probability of each solution. After selecting any SIV of that solution for 


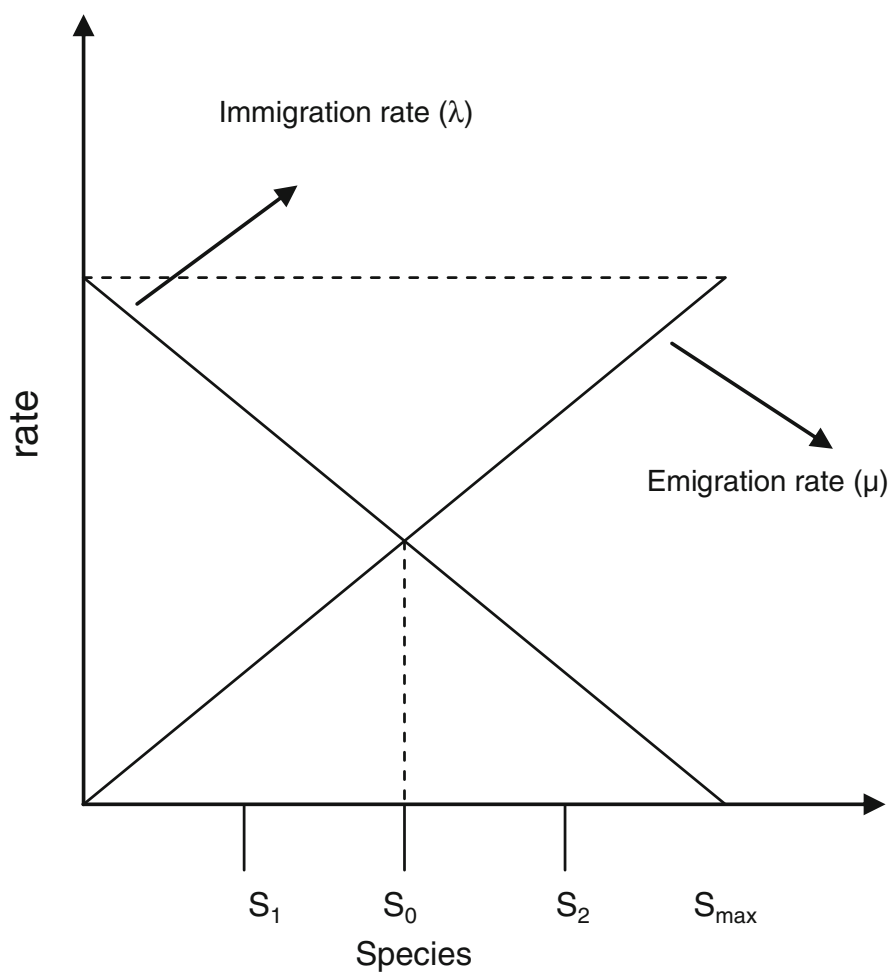

Figure 2. The relationship of fitness of habitats.

modification, emigration rates $\mu_{j}$ of other solutions $\mathrm{S}_{j}\left(\mathrm{~S}_{j}\right.$ is $\mathrm{j}^{\text {th }}$ solution set other than $\mathrm{Si}$, i.e., $\mathrm{j}=\mathrm{i}$ ) will be determined. Then based on probability, one solution $\mathrm{S}_{j}$ among the population set will migrate randomly to chosen SIVs to the selected solution $\mathrm{Si}$.

\subsection{Mutation}

Mutation rate of each set of solutions can be calculated in terms of species count probability. Each species count probabilities can be calculated using the differential equations referred in Simon (2008). Each habitat member has an associated probability. The solution with low probability is likely to mutate. The solution with highest probability has the elitism of not mutated or migrated. Similarly, higher probability solutions have rare chance to mutate.

\subsection{Algorithm for biogeography based side lobe level reduction}

Step 1: Initialize the parameters

Number of variables (NumVar), Number of islands (popsize), Maximum generations (MaxGen),

Mutation rate, Elite count, Species count.

Step 2: Define immigration rate and emigration rate.

Step 3: Generate randomly, n-islands -the population.

Step 4: Calculate the immigration rate and emigration rate for each island.

Step 5: Calculate the immigration probability and emigration probability of each island. 
Step 6: Select one island for immigration and another one for emigration based on their probability.

Step 7: Migrate randomly selected SIVs based on the selected islands in the previous step.

Step 8: Probabilistically perform mutation based on the mutation probability for each island.

Step 9: Calculate the side lobe level (fitness) of each individual island. Use the following steps

(i) initialize the parameters : Element spacing, Number of elements.

(ii) define Theta space, define the array factor, steering vector.

(iii) calculate the array factor and choose the weight vector which gives the $\mathrm{SLL}<-40 \mathrm{~dB}$.

Step 10: If the termination criterion is not met, go to step 3; otherwise, terminate.

\subsection{Linear migration model}

Dan Simon developed BBO with linear migration model only. The immigration rate $\lambda_{k}$ and emigration rate $\mu_{k}$ are linear functions of species count ' $\mathrm{k}$ ' in the habitat or island. The mathematical equation of this model is given by (4) and (5) and illustrated in figure 2. At equilibirium $\mathrm{K}_{o}$ and its probability $\mathrm{P}_{k_{o}}$ are given by (6) and (7)

$$
\begin{gathered}
k_{0}=\frac{n I}{I+E}, \\
P_{k_{0}}=\frac{\left(\frac{I}{E}\right)^{k_{o}}\left(\frac{n !}{k_{0} !\left(n-k_{0}\right) !}\right)}{1+\sum_{i=1}^{n}\left(\frac{I}{E}\right)^{i}\left(\frac{n !}{i !(n-i) !}\right)} .
\end{gathered}
$$

Immigration rate and emigration rate have inverse relationship. Emigration rate is high for a highly crowded island and the immigration rate will be less. For this model, the probability $P_{k}$ is approximated to a Gaussian function as shown in figure 3.

\subsection{Sinusoidal migration model}

Sinusoidal migration model is proposed and analysed using benchmark functions in Ma (2010) and proved to be the most promising model for engineering application.

$$
\begin{gathered}
\lambda_{k}=\frac{I}{2}\left(\cos \left(\frac{k \pi}{n}\right)+1\right), \\
\mu_{k}=\frac{E}{2}\left(-\cos \left(\frac{k \pi}{n}\right)+1\right), \\
K_{0}=\frac{n}{\pi} \cos ^{-1}\left(\frac{E-I}{E+I}\right), \\
P_{k_{0}}=\frac{\prod_{j=1}^{k_{0}}\left(\frac{I}{E}\right)^{k_{o}}\left(\frac{\sin ^{2}\left(\frac{n+j-1}{2 n} \pi\right)}{\sin ^{2}\left(\frac{j}{2 n} \pi\right)}\right)}{1+\sum_{i=1}^{n} \prod_{j=1}^{i}\left(\frac{I}{E}\right)^{i}\left(\frac{\sin ^{2}\left(\frac{n+j-1}{2 n} \pi\right)}{\sin ^{2}\left(\frac{j}{2 n} \pi\right)}\right)} .
\end{gathered}
$$




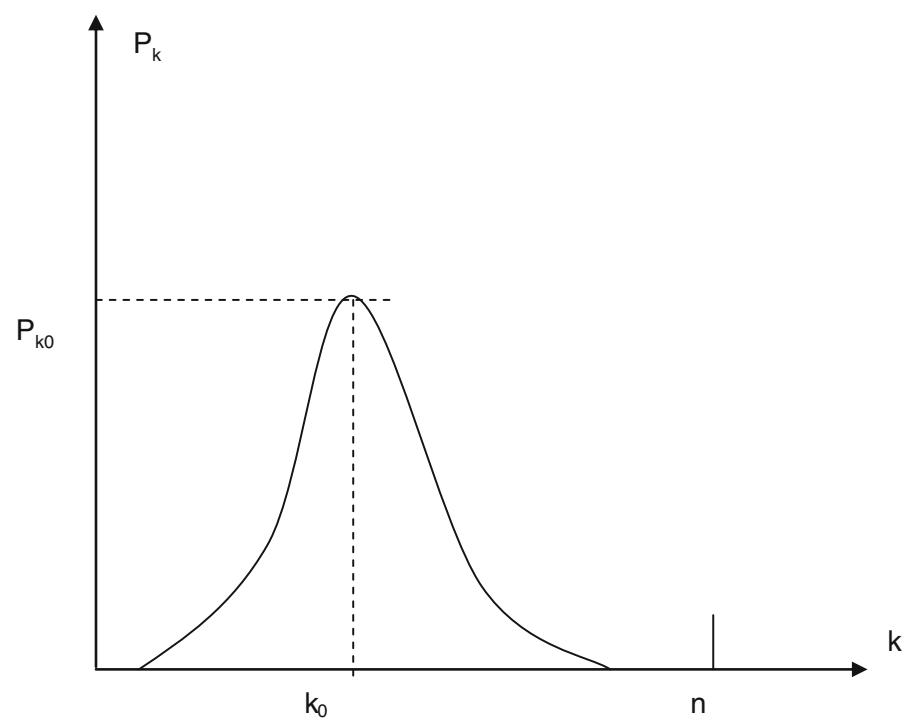

Figure 3. Steady state probability as a function of species count for Linear migration model.

The idea of introducing this sinusoidal migration model in BBO is proposed in our paper to optimize the side lobe level reduction. The immigration rate and the emigration rates are given by (8) and (9), respectively. The same is illustrated in figure 4 . When the number of species increase, the immigration rate linearly decreases because the habitat becomes more crowded and

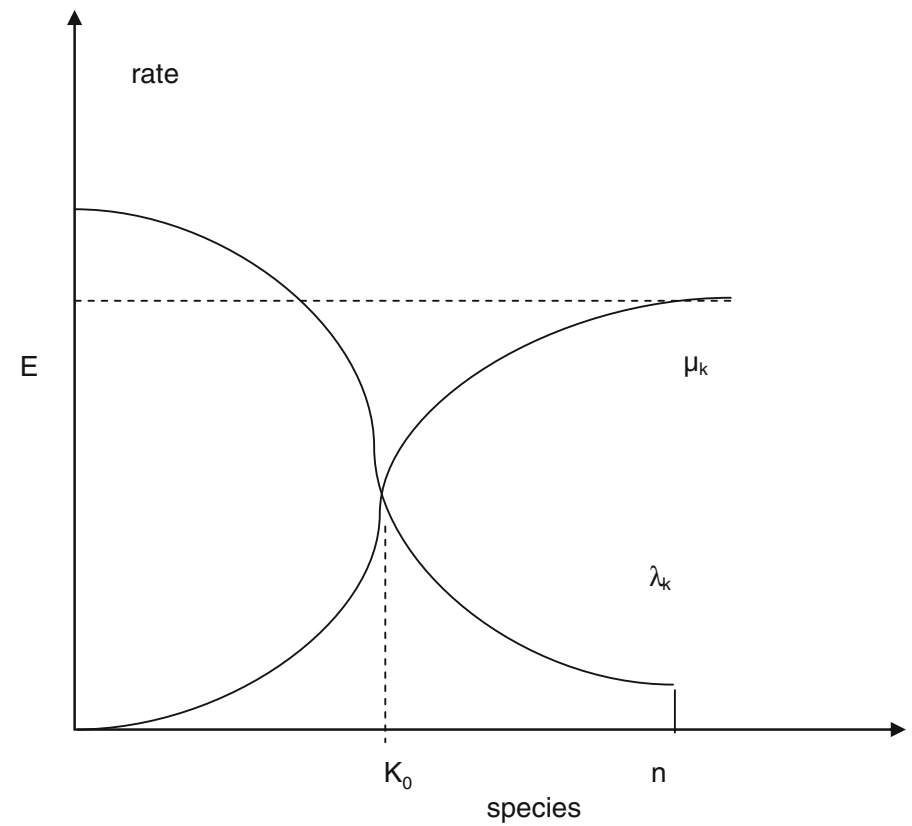

Figure 4. Sinusoidal migration model showing immigration and emigration rates. 


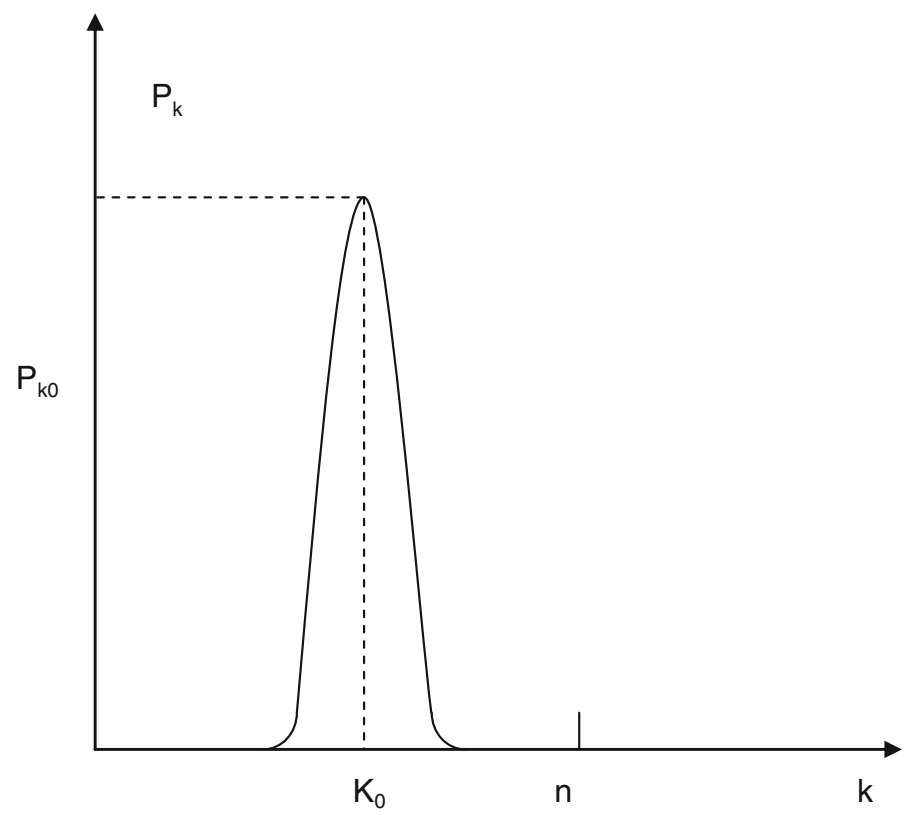

Figure 5. Steady state probability as a function of species count for Sinusoidal migration model.

fewer species are able to successfully survive immigration to the habitat, while the emigration rate linearly increases because more species are able to leave the habitat to explore other possible residences. At equilibrium, $\mathrm{K}_{o}$ and its probability $\mathrm{P}_{k_{o}}$ are given by (6) and (7). For this model, the probability is an even function with respect to its midpoint, defined by (11) and shown in figure 5 .

\section{Simplified BBO (S-BBO)}

Simplified BBO evaluates the fitness of each habitat and the best fitted habitat with high HSI $\left(\mathrm{H}^{2} \mathrm{SI}\right)$ is considered as the emigrating island. Independent of its fitness any SIV can be selected
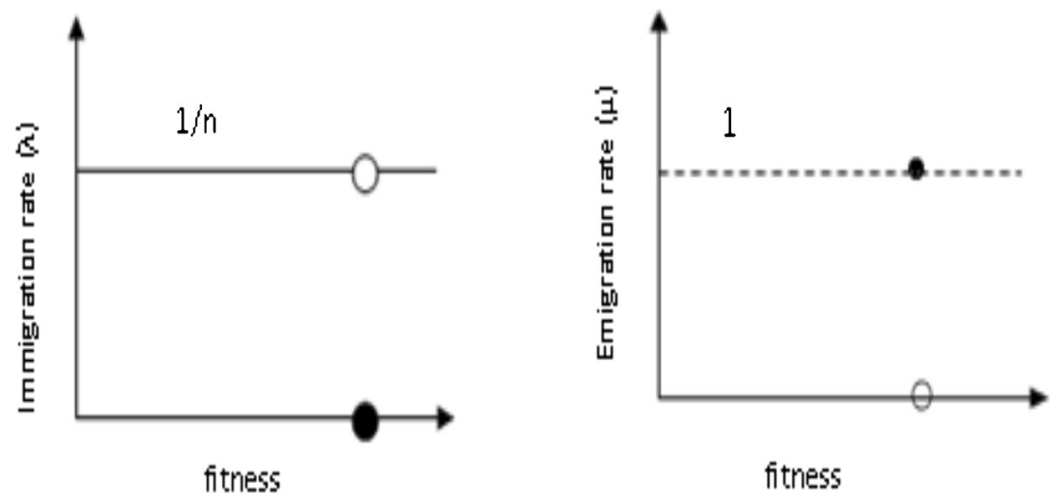

Figure 6. Migration curves of Sinusoidal migration model. 
as immigrant island other than the one with $\mathrm{H}^{2} \mathrm{SI}$. The migration curves of SBBO are modified such that they have uniform probability distribution. The immigration rate of every SIV is $1 / n$ where ' $n$ ' is the number of SIVs in an island. The emigration rate is 1 for the best fitted $\mathrm{H}^{2} \mathrm{SI}$ and o for the rest. The curves are shown in figure 6 . The algorithm is given below as

Step 1: Calculate the fitness of all SIVs.

Step 2: Find the best fitted solution $\left(\mathrm{H}^{2} \mathrm{SI}\right)$. Name this as the emigration island $\mathrm{S}_{i}$.
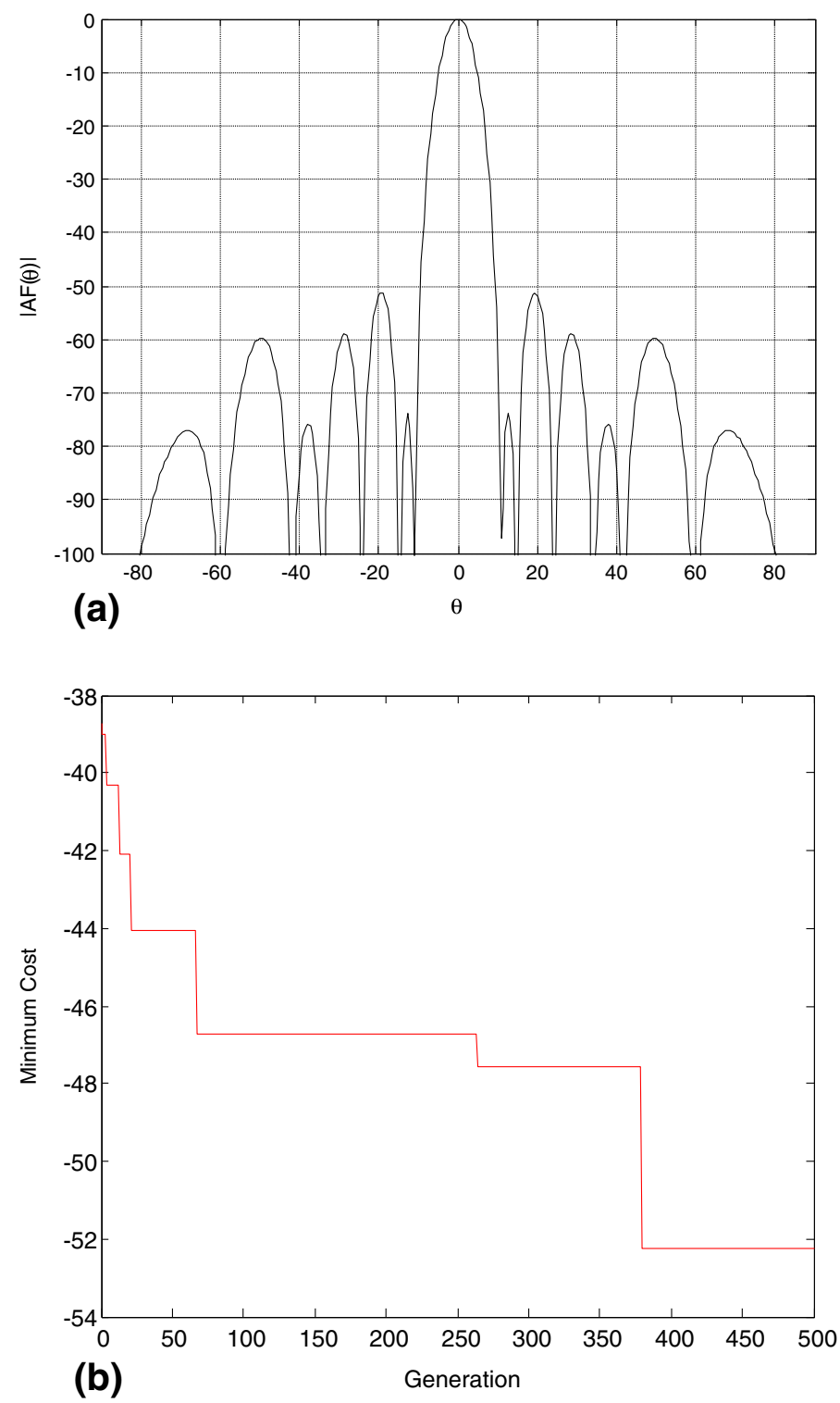

Figure 7. Optimized radiation pattern and convergence curve for BBO-Sinusoidal migration model for $\mathrm{N}=16$ elements. 
Step 3: Randomly select any SIV other than $\mathrm{H}^{2}$ SI. Refer this as immigration island $\mathrm{S}_{j}$.

Step 4: Migrate $S_{i}$ to $S_{j}$.

\section{Performance of BBO on side lobe level reduction using sinusoidal migration model}

The simulation experiments are performed in MATLAB using Intel Pentium dual core processor of $1 \mathrm{~GB}$ memory.

\section{Experiment 1:}

The following parameters are used in this simulation experiment

The population size: 50; Maximum generations: 500; Number of variables: 8

The problem dimension: 16; The best and mean SLL obtained out of 500 generations are $-52.26 \mathrm{~dB}$ and $-29.1448 \mathrm{~dB}$, respectively. The execution time is $18.218 \mathrm{~s}$. The diversity measure is 6942 . It converges to $-40 \mathrm{~dB}$ in less than 2 generations and to -52.26 in 375 generations. This is shown in figures 7(a) and (b).

\section{Experiment 2:}

The following parameters are used in this simulation experiment

The population size: 50; Maximum generations: 500; Number of variables: 12

The problem dimension: 24; The best and Mean SLL obtained are $-54.3282 \mathrm{~dB}$ and $-32.7887 \mathrm{~dB}$.

Diversity measure is 7204 and execution time is $21.153 \mathrm{~s}$.

The performance of BBO-linear migration model and BBO-sinusoidal migration model are compared which is illustrated figure 8 . BBO-sinusoidal migration model performs better than linear migration model.

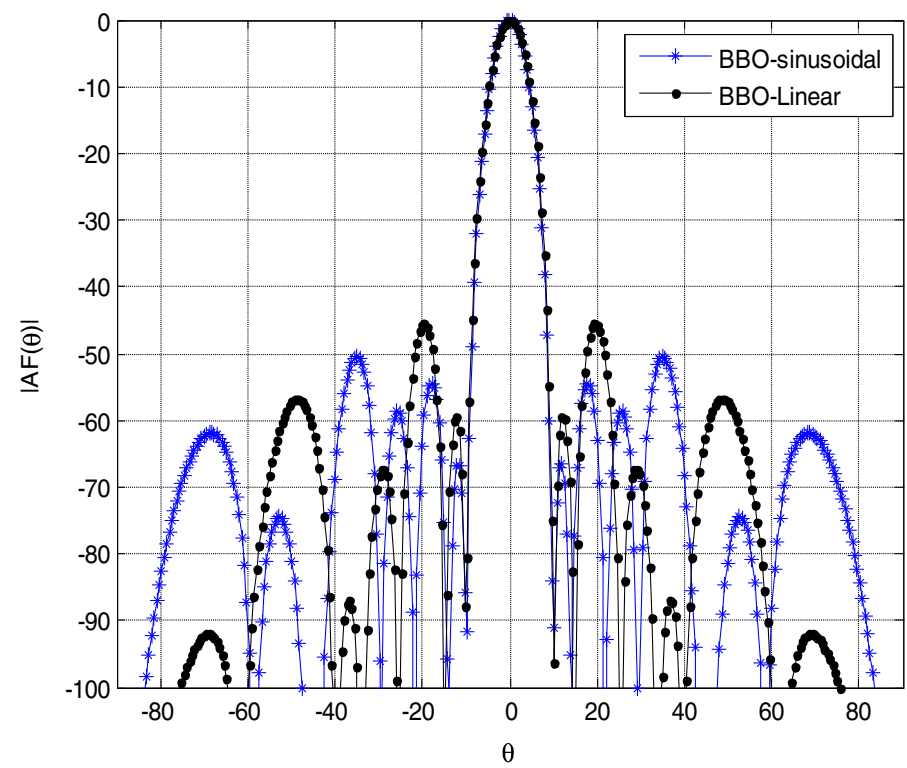

Figure 8. Comparing optimized radiation patterns of BBO models - Sinusoidal and Linear Migration. 


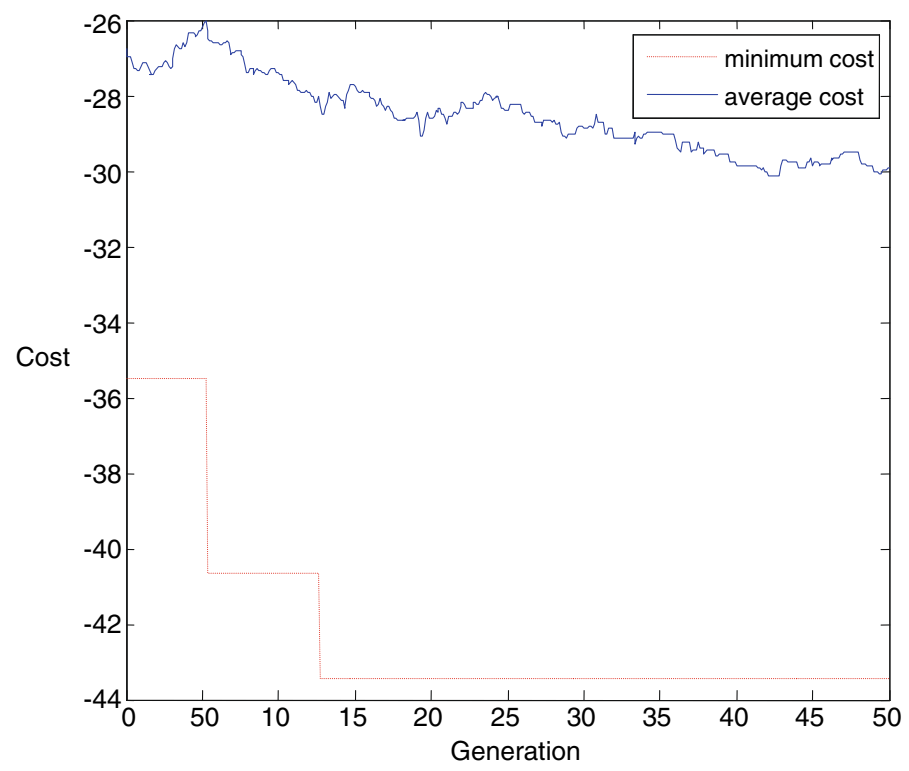

Figure 9. Convergence curve for $\mathrm{N}=16$ elements.

\section{Performance of S-BBO on side lobe level reduction}

Two experiments are discussed here.

Experiment 1:

The following parameters are used in this simulation experiment

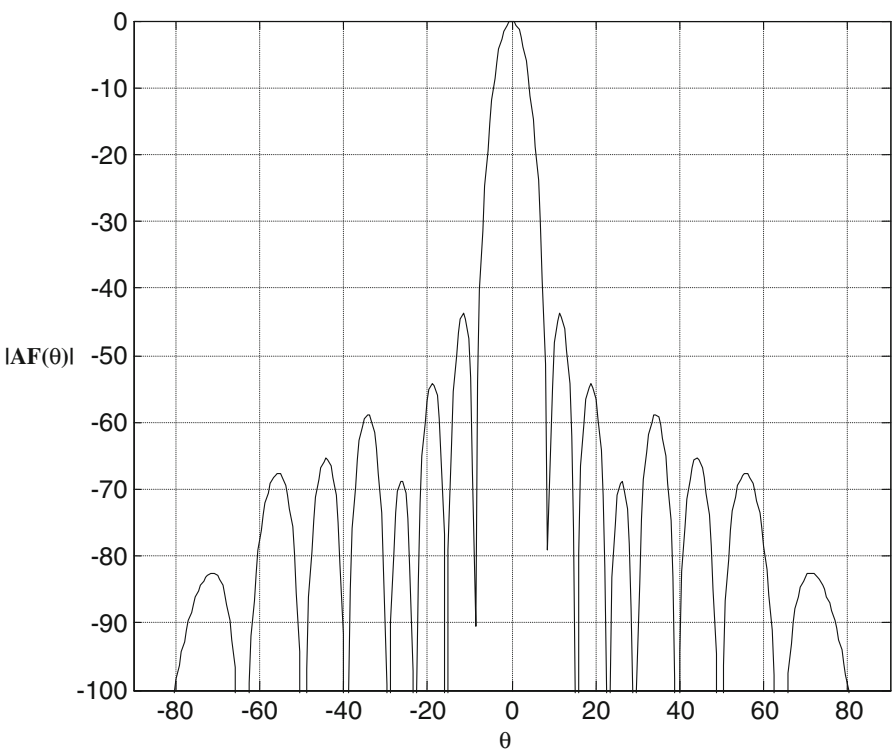

Figure 10. Optimized radiation pattern for $\mathrm{N}=16$ elements with $\mathrm{S}-\mathrm{BBO}$. 


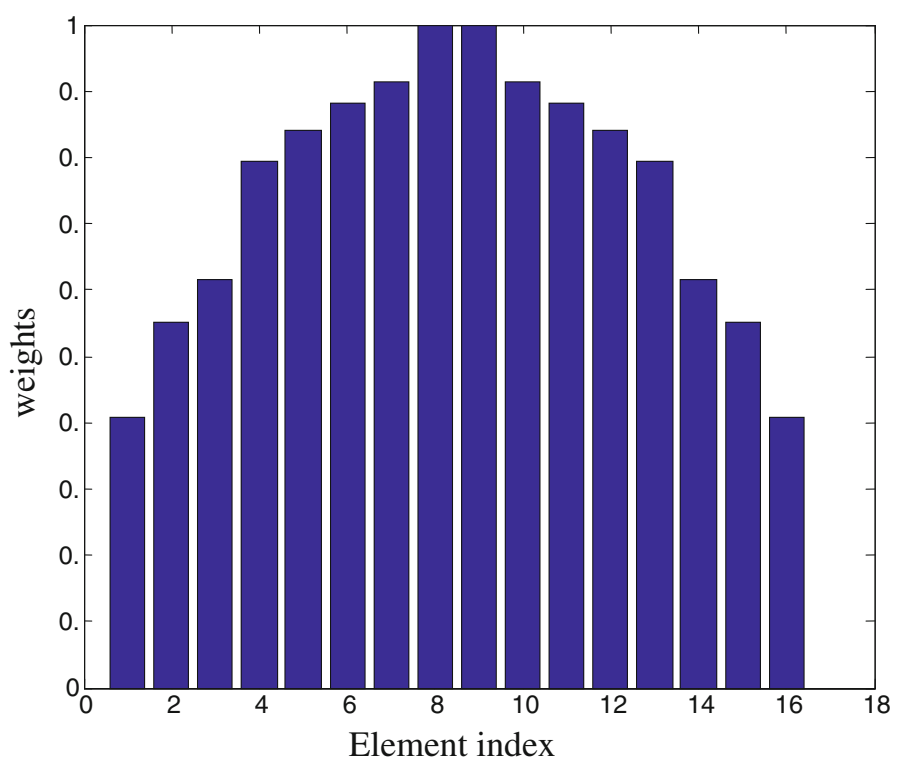

Figure 11. Amplitude excitations for $\mathrm{N}=16$ elements with $\mathrm{S}-\mathrm{BBO}$ model.

The population size: 50; Maximum generations: 500; Number of variables: 8

The problem dimension: 16 . The program completes the run generating the best SLL and mean SLL out of 500 generations as $-43.4538 \mathrm{~dB}$ and $-29.943 \mathrm{~dB}$. This is shown in the convergence curve of figure 9 and the radiation pattern of figure 10 with the SLL of $-43.4538 \mathrm{~dB}$. Figure 11 shows the weight vector or the amplitude excitations. It takes 125 generations to converge. There are 46 unique solutions in final population. Among 500 generations, there are improvements in generations 53 and 74 with an improvement amount of $51.7 \%$ and $28 \%$. The execution time taken is $5.428514 \mathrm{~s}$.

Experiment 2:

The population size: 50; Maximum generations: 500; Number of variables: 12; The problem dimension: 24; The best SLL and mean SLL out of 500 generations are $-49.3559 \mathrm{~dB}$ and $-30.292 \mathrm{~dB}$. It takes 330 generations to converge. There are 50 unique solutions in final population. Improvement is achieved in $333^{\text {rd }}$ generation with an improvement amount of $45 \%$. The corresponding weight vector is [0.0436 0.0741 0.1300 0.15340.1798 0.24470.40120.4408

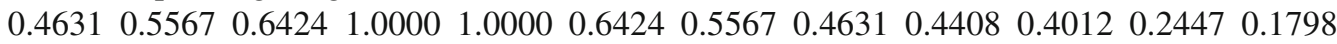
$0.15340 .13000 .07410 .0436]$. The execution time taken to run this simulation is $4.638468 \mathrm{~s}$.

Table 1. Performance of S-BBO with $\mathrm{N}=8,16,20$ and 24 elements.

\begin{tabular}{lllll}
\hline $\begin{array}{l}\text { No. of } \\
\text { Elements }\end{array}$ & HPBW(max) & Max BWNN & Maximum no. of side lobes & Mean execution time (s) \\
\hline 8 & 10.8 & 44 & 6 & 4.76 \\
16 & 5.54 & 23 & 10 & 5.36 \\
20 & 4.7 & 18.5 & 10 & 5.21 \\
24 & 3.6 & 15 & 22 & 5.58 \\
\hline
\end{tabular}




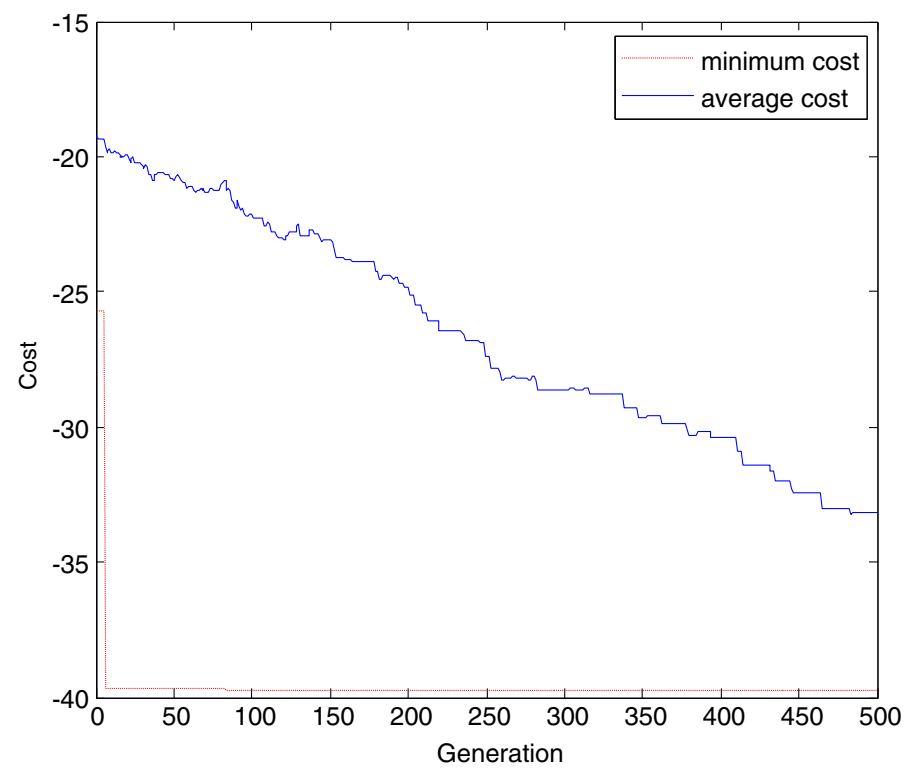

Figure 12. Convergence curve for $\mathrm{N}=8$ elements.

The performance of S-BBO is analysed with different number of antenna elements $\mathrm{N}=8,16$, 20 and 24 elements which is tabulated in table 1.

As the number of elements increases

- The beamwidth decreases that is, the directivity increases.

- Reyleigh resolution limit decreases (i.e., $\mathrm{BW}_{\mathrm{NN}}$ ).

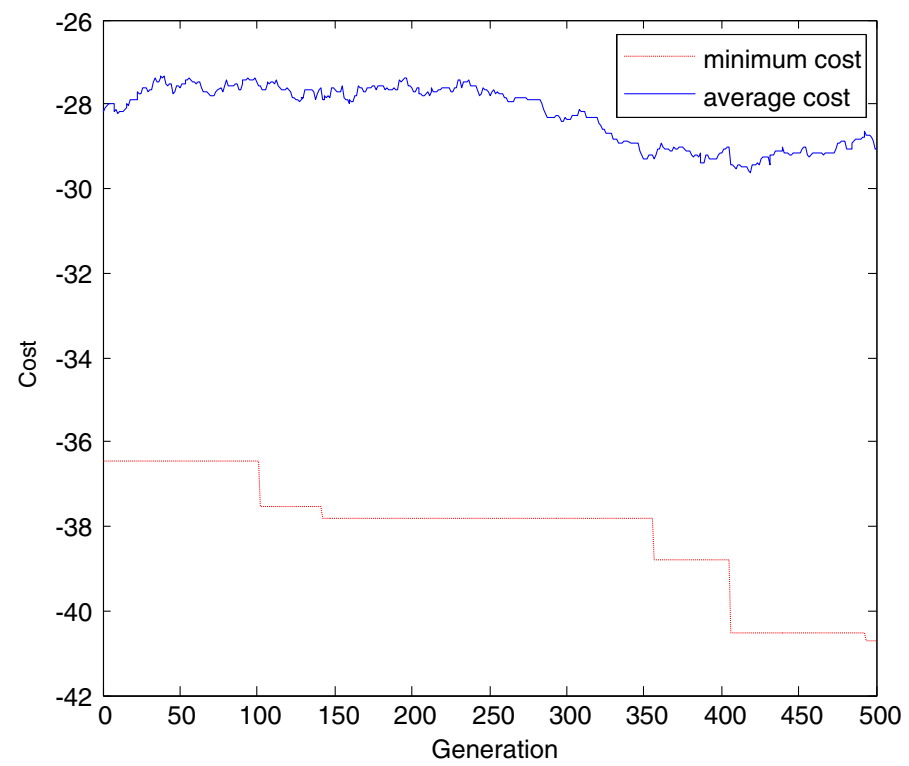

Figure 13. Convergence curve for $\mathrm{N}=16$ elements. 


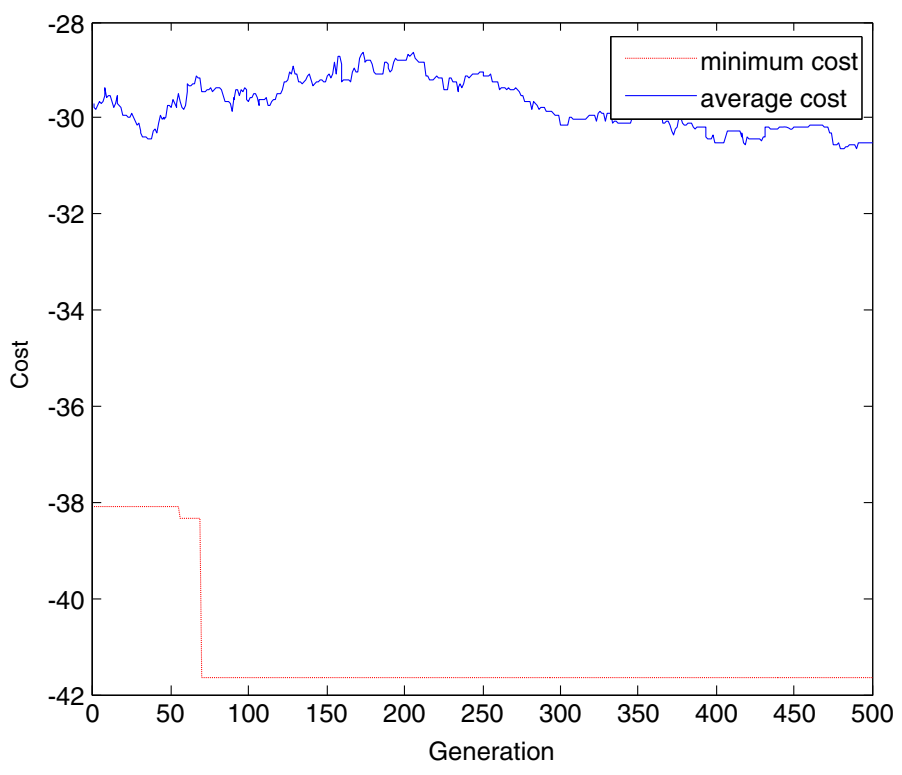

Figure 14. Convergence curve for $\mathrm{N}=20$ elements.

- The number of sidelobes increases which means waste of power in unwanted direction.

- The execution time increases.

The designer may choose any antenna array based on his needs considering the above trade-offs.

Based on the convergence curves of S-BBO for 8, 16 and 20 number of elements, the following observations are made.

- There is vast difference between the average and best cost incase of large number of elements. The difference gets reduced as the number of elements decreases. This can be clearly seen by comparing figures $12-14$.

- For small number of elements, convergence is fast at the earlier stage itself and no improvement later on. But the average cost value increases. We are not able to achieve the best solution.

- Total execution time increases as number of elements increase. The best solution is achieved in later generations. These findings are tabulated in table 1.

Table 2. Performance comparison for different EAs.

\begin{tabular}{lllll}
\hline EA & HPBW $(\mathrm{deg})$ & $\mathrm{BW}_{\mathrm{NN}}(\mathrm{deg})$ & Max no. of side lobes & Execution time (s) \\
\hline S-BBO & 5.54 & 23 & 10 & 5.36 \\
BBO -linear & 7.2 & 32 & 12 & 19.52 \\
BBO -sinusoidal & 5.12 & 20 & 14 & 18.06 \\
ACO & 5.87 & 26.62 & 10 & 22.03 \\
GA & 5.12 & 20.75 & 14 & 22.96 \\
Stud GA & 5.25 & 21.5 & 14 & 23.47 \\
\hline
\end{tabular}


Table 3. Performance comparison of different EAs for SLL.

\begin{tabular}{lll}
\hline EA & Best minimum SLL $(\mathrm{dB})$ & Mean SLL $(\mathrm{dB})$ \\
\hline S-BBO & -44.32 & -28.94 \\
BBO -linear & -51.48 & -28.45 \\
BBO -sinusoidal & -53.26 & -28.58 \\
ACO & -47.98 & -29.4 \\
GA & -48.22 & -30.66 \\
Stud GA & -49.36 & -26.54 \\
\hline
\end{tabular}

\section{Comparative performance analysis of $\mathrm{S}-\mathrm{BBO}$ with $\mathrm{BBO}$-linear migration, BBO-sinusoidal migration, ACO, GA and stud GA}

For the comparative performance of different EAs, 16 number of elements are considered. The program is run for 25 times and the mean values are calculated. The performance measures such as Half Power Beamwidth(HPBW), Beamwidth between first nulls $\left(\mathrm{BW}_{N N}\right)$, total number of side lobes $(\max )$ and the time taken for completion of the program execution are considered. It is tabulated in table 2 .

The minimum side lobe level achieved for the EAs considered in our work, are tabulated in table 3.

\section{Conclusion}

This paper discusses the general theory of BBO and S-BBO. We have also proposed the application of the above techniques for reducing the side lobe level to a maximum. The influence of the two migration models with BBO are also explored. S-BBO on ULA synthesis is investigated. Experimental results clearly show that the sinusoidal migration model of BBO results in producing optimum weight vector values in performance. S-BBO outperforms all others in terms of minimum execution time. A comparative study with other EAs like ACO, GA, and StudGA was also performed. The effect of number of variables - the dimension and the convergence of SBBO are analysed. Further, this work can be extended to constrained optimization problems and multi-objective optimization problems for different array geometries with adaptive migration rates and other migration models.

\section{References}

Constantine A Balanis 2005 Antenna Theory: Analysis and Design $3^{\text {rd }}$ Edition, John Wiley \& Sons Dan Simon 2008 Biogeography-Based Optimization, IEEE Transactions on Evolutionary Computation. 12(6)

Dan Simon 2011 A probabilistic analysis of a simplified biogeography-based optimization algorithm. Evolutionary Computation Summer 19(2): 167-188

Randy L Haupt and Douglas H Werner 2007 Genetic Algorithms in Electromagnetics, IEEE PRESS, WileyInterscience

Haiping Ma 2010 An analysis of the equilibrium of migration models for biogeography-based optimization. Information Sciences 180: 3444-3464

Singh U, Kumar H and Kamal T S 2010 Linear array synthesis using biogeography based optimization. Progress Electromagnetics Res. M 11: 25-36 